PACS 61.

\title{
Formation of martensite crystals in the limiting case of a supersonic growth rate
}

\author{
M.P. Kashchenko ${ }^{1,2 \dagger}$, V. G. Chashchina ${ }^{1,2}$ \\ †mpk46@mail.ru
${ }^{1}$ Ural Federal University named after the first President of Russia B. N. Yeltsin, 19 Mira str., 620002, Ekaterinburg, Russia
${ }^{2}$ Ural Federal University, 37 Sybirskyi Tract, 620100, Ekaterinburg, Russia

\begin{abstract}
A brief overview of the basic concepts and achievements of the dynamic theory of supersonic growth of martensite crystals is given. It is shown that a consideration of the limiting case of a high growth rate allows for achievement of the physical transparency and mathematical simplicity of the description. Attention is focused on a number of topical problems of a new stage of investigations.
\end{abstract}

Keywords: martensitic transformations, the initially excited state, supersonic rate.

\section{Introduction}

The present review is devoted to the limiting case of martensitic transformations (MT) proceeding with strongly marked signs of first-order transitions between lattice states with symmetries not connected by a co-subordinate relationship. A classic example is the $\gamma-\alpha \mathrm{MT}$ in iron-based alloys. The progress achieved to date allows to speak about constructing a dynamic theory of lamellar crystal formation with a supersonic rate in original austenite regions, exceeding a certain critical size and free from defects. Although the relevant information is presented in monographs [1-3], available in open access, it is useful to provide, for beginning researchers, a brief summary of the ideology that is the essence of the paradigm, significantly differing from the traditional versions based on the quasi-equilibrium approach. In other words, our aim is to outline the shortest quality path to understanding the physical mechanism for realization of MT, taking the reader to the forefront of the research, avoiding a retrospective coverage of the issue which is of interest on its own, but is poorly compatible with a concise rendering. Nevertheless, it is impossible not to note that the research topic of MT entered the mainstream of modern science after the work [4] where the diffusionless character of MT was established (see also [5]).

It is worth reminding that the value of any limiting case is the possibility of a noticeable simplification of a description, which is important not only from the physical point of view, as it allows to focus attention on the dominant factors, but also in the methodological aspect, as it opens up an opportunity for formulation of the simplest mathematical model reflecting, nevertheless, the most important features of a complex picture.

For instance, in our case a comprehension of the fact of the supersonic rate of crystal growth leads to serious simplifications of the description, since it allows for dissociation from the models of interphase boundary mobility based on the notions of moving dislocations ${ }^{1}$, focusing our attention exclusively on the wave control of the process of martensite crystal growth.

A discussion of the results of the measurements of the martensite crystal growth rate during $\gamma-\alpha \mathrm{MT}$, conducted in $[6,7]$, are presented in item 1.2 of the monograph [1]. The results presented in [8] also agree with the data given in $[6,7]$.

\section{The basic models of heterogeneous nucleation and wave growth of martensite crystals}

A high growth rate, in accordance to the logic of the study, immediately brings up a question about the specifics of the initial state of transformation. Indeed, the primal character of transformation dictates the necessity of a heterogeneous nucleation, i.e. an explicit participation of defects, and the wave mechanism of growth demands to allow for the emergence of the initially excited (vibrational) state of the lattice in the elastic field of a defect, from which the controlling wave process (CWP) starts. It is apparent that the construction of a quasi-equilibrium nucleus, with the help of isolation of a certain region of the parent phase with configured sets of dislocation loops, is not required here, which is again an evident simplification in the task formulation, although it requires knowledge of the elastic fields typical for the parent phase of defects (mainly, dislocations). But the calculations of these fields for actual anisotropic crystals are to a great extent standardized and

\footnotetext{
${ }^{1}$ Models with "transformation dislocations" are acceptable for the description of another limiting case - the thermoelastic realization of MT, close to second-order transitions.
} 
therefore do not cause any significant difficulty. Note should be made that a search for special defect configurations (as nucleation regions) has not yielded any positive results, while the process of initiating a fast growth is typical for both single crystals (nevertheless, containing dislocations) and large austenite grains. Therefore it seems very probable that individual dislocations (typical for the lattice symmetry of the parent phase) play the role of dislocation nucleation centers (DNC) that distort with their elastic fields the symmetry of the parent phase, marking the regions most favorable for the emergence of the initially excited state. This qualitative motivation leads to the scheme displayed in Fig. 1 presenting a section (with sizes $d_{1,2}$ ) of a strategically deformed volume in the form of a rectangular parallelepiped built on its own vectors $\xi_{\mathrm{i}}$ of the elastic strain field tensor of the DNC.

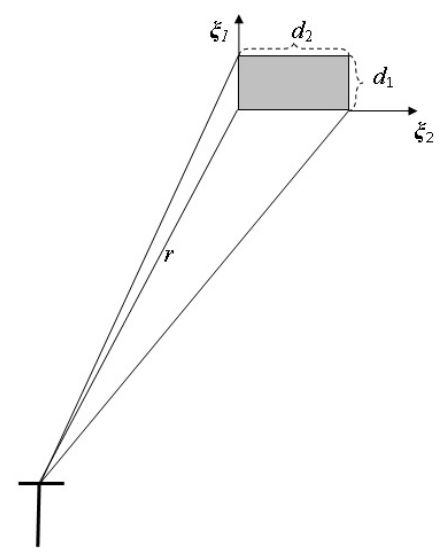

Fig. 1. Localization region of the initially excited state in the elastic field of a separate dislocation: the correlation between the scales $r$ and $d$.

Notably, there are angle ranges $\Delta \Theta$ (in the vicinity of a certain value $\Theta_{0}$ ) with strains of the opposite sign along the axes $\xi_{1,2}$ orthogonal to the largest edge $\xi_{3}$, along which the strain is close to the zero value. The corresponding main values of the strain tensor in the nucleation region not only satisfy the condition of plane strain with a pair of invariant (slightly distorted) planes:

$$
\varepsilon_{1}>0, \quad \varepsilon_{2}<0, \quad \varepsilon_{3} \approx 0,
$$

but also $\varepsilon_{1}$ and $\left|\varepsilon_{2}\right|$ are close to the maximum values $\varepsilon_{1}\left(\Theta_{0}\right)$, $\left|\varepsilon_{2}\left(\Theta_{0}\right)\right|$ (the extrema are searched by the angle variable $\Theta$ at a certain fixed distance $r$ to the dislocation line). Consequently, in the region of nucleation the defect's elastic field reduces in the maximum degree the energy barrier for the onset of plane strain with an invariant (at $\varepsilon_{3}=0$ ) or slightly distorted (at $\varepsilon_{3} \approx 0$ ) plane.

It is clear that the elastic field of the defect in the parallelepiped's section (on scale $d$ ) is approximately uniform, in case the following condition is fulfilled:

$$
d / r \leq 0.1
$$

Suppose that the average distance between the dislocations is equal to $L$. Then the influence of other dislocations can be neglected, if

$$
r \sim 0.1 L
$$

It follows from (2) and (3) that

$$
d \sim 10^{-2} \mathrm{~L}
$$

i.e. between the spatial scales $d$ and $L$ there is a relation, important for understanding the observed spatial effects (see the discussion in [3]). It is important that during the transition to fine grains the role of $L$ is played by the grain diameter $D$.

It is natural to believe that the role of the volume element in which the transition of an atom collective through the energy barrier (with energy release and excitation of vibrations) is realized, is played by a three-dimensional cell in the form of an elongated rectangular parallelepiped. Its faces vibrate in pairs in the opposite phase, stimulating threshold deformation of the tension-compression type in mutually orthogonal directions coinciding with the directions of propagation of wave beams radiated by the vibrating parallelepiped. The lattice sequentially loses its stability, forming a plate-like region there, where wave beams superimpose with atomic displacement fields favorable for realization of threshold deformation (see Fig.2).

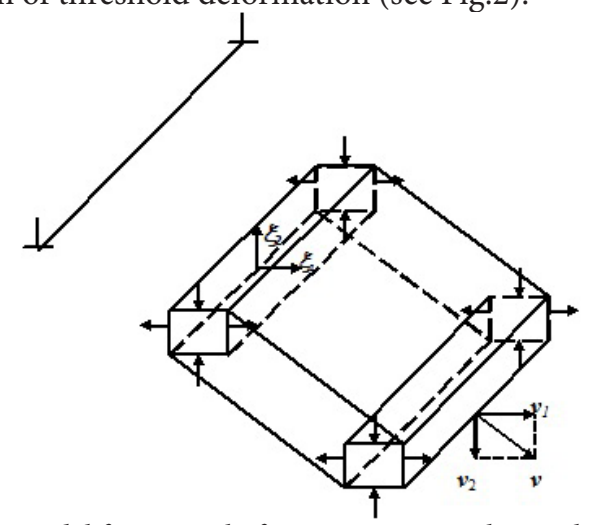

Fig. 2. Wave model for control of martensite crystal growth.

The formation of the plate-like prototype of a martensite crystal can be naturally interpreted as the movement of the parallelepiped with a velocity $\mathrm{v}$, representing the vector sum of the velocities $v_{1}$ and $v_{2}$ of the wave beams. Since the energy release required for the autocatalytic feeding of a wave takes place only in the volume that has experienced threshold deformation, it is the supersonic rate $v$ that becomes the actual crystal growth rate.

Consequently, a synthesis of the concepts of heterogeneous nucleation and wave growth is achieved if we consider that the wave normals $n_{1}$ and $n_{2}$ of the wave beams which describe in the superposition region the tensile strain $\left(\varepsilon_{1}>0\right)$ and the compression strain $\left(\varepsilon_{2}<0\right)$, respectively, are collinear to their own vectors $\xi_{i}(i=1,2)$ of the strain tensor of the defect elastic field in the nucleation region:

$$
n_{1}\left\|\xi_{1}, \quad n_{2}\right\| \xi_{2}, \quad n_{1} \perp n_{2} \quad\left|n_{i}\right|=\left|\xi_{i}\right|=1
$$

Hence, the CWP inherits the information about the directions of the principal strain axes. It is easy to show [1] that the normal $N_{w}$ to the habit plane, connected with the propagation of the CWP, is given by the kinematic relation:

$$
N_{\mathrm{w}} \| n_{2}-n_{1} æ, \quad æ=\left(v_{2} / v_{1}\right),
$$


where $v_{1}$ and $v_{2}$ are the velocity moduli of wave propagation in the directions $n_{1}$ and $n_{2}$. On the other hand, in the case of uniform plane deformation of tension-compression, the normals to invariant planes take the following form:

$$
\begin{gathered}
N_{\mathrm{d}}=\xi_{2} \pm k \xi_{1} \\
k=\frac{1-\left|\varepsilon_{2}\right|}{1+\varepsilon_{1}}\left(\frac{\varepsilon_{1}}{\left|\varepsilon_{2}\right|} \frac{\left(2+\varepsilon_{1}\right)}{\left(2-\left|\varepsilon_{2}\right|\right)}\right)^{\frac{1}{2}}
\end{gathered}
$$

Since the CWP carries strain with an invariant plane, it is natural to believe that there takes place a coincidence of the kinematic and dynamic descriptions of the habit plane during the CWP propagation, i.e. formulas (6) and (7) describe the same habitus if the values of $\varepsilon_{i}$ in (8) correspond to the threshold values. Then, taking into account (5), the following important condition is obtained:

$$
æ=k \text {, }
$$

describing the relation between strains and wave velocities that exists during the CWP propagation.

It has been established experimentally that the application of external elastic stresses considerably displaces the temperature $M_{\mathrm{s}}$ of the start of MT. This means that the threshold strains $\varepsilon_{\text {th }}$ lie in the elastic range of values. Since $\varepsilon_{\mathrm{th}}<<1$, condition (9) takes the following form:

$$
æ=\frac{v_{2}}{v_{1}}=k \approx \sqrt{\frac{\varepsilon_{1}}{\left|\varepsilon_{2}\right|}}
$$

where the velocities $v_{2}$ and $v_{1}$ can be calculated with the help of the Christoffel equation [9], using the elasticity moduli of the initial crystal lattice (preferably at the temperature $M_{\mathrm{s}}$ ).

Let us make a few remarks.

1. Taking into consideration the principal significance of the notions of the emergence of the initially excited state, experiments were performed [10-13] on its physical modeling. Laser pulses (with a duration of $20 \mathrm{ps)} \mathrm{were} \mathrm{used,}$ with a close to linear shape of the trail affecting the surface of specially oriented single crystals. The results confirmed the expected initiation of martensite crystal growth.

2. The described algorithm for calculation of habit plane orientations allows to identify the DNC of specific martensite crystals on the basis of the requirement for the estimated values of $N_{w}$ to coincide with the observed ones.

3. The smallness of threshold strains allows to use in the analytical description the harmonic form of recording the displacements (and strains) of the lattice in the localization region of the initially excited state (see [14] and chapter 2 in [2]). Such an approximation makes it possible, at the prescribed values of $\varepsilon_{\text {th }}$, to find easily the transverse size $d_{1,2}$ of the localization region of the initially excited state as quite definite fractions of half-waves $\lambda_{1,2} / 2$ correlated to wave beams being part of the CWP (the inequality $d_{1,2}<\lambda_{1,2} / 2$ must necessarily be fulfilled).

4. The simplest system of wave equations reflecting the interaction between two beams with orthogonal wave vectors (along $\mathrm{x}$ - and $\mathrm{y}$-directions) is also discussed in [2] and [14]. In the context of the strains $\varepsilon_{1,2}$ in $x$ - and $y$-directions this system has the following form:

$$
\left\{\begin{array}{l}
\dot{\varepsilon}_{1}+v_{1} \varepsilon_{1}^{\prime}+b_{1}\left(\varepsilon_{1}, \varepsilon_{2}\right) \varepsilon_{1}=0 \\
\dot{\varepsilon}_{2}+v_{2} \varepsilon_{2}^{\prime}+b_{2}\left(\varepsilon_{1}, \varepsilon_{2}\right) \varepsilon_{2}=0
\end{array},\right.
$$

where $\quad \dot{\varepsilon}_{1,2} \equiv \frac{\partial \varepsilon_{1,2}}{\partial t}, \varepsilon_{1}^{\prime}=\frac{\partial \varepsilon_{1}}{\partial x}, \varepsilon_{2}^{\prime}=\frac{\partial \varepsilon_{2}}{\partial y}$ and the interaction is reflected by the introduction of the "effective" wave attenuations $b_{1,2}$.

It should be taken into consideration that the parameters $b_{1,2}$ are positive in the case of the absence of the amplification mechanism, but they satisfy the condition $b_{1,2} \leq 0$ if the condition of wave generation in the region of application of controlling waves with the strains $\varepsilon_{1}>0$ and $\varepsilon_{2}<0$ is fulfilled. It is evident that the simplest case of the amplification and loss compensation corresponds to $b_{1,2}=0$. In reality, there will be attenuation compensation only in the region of energy release, therefore the interaction of waves is reflected in system (11) through the dependencies of the coefficients $b_{1,2}$ on the strains $\varepsilon_{1,2}$. In the simplest case, it is convenient to present these dependencies in the following form:

$$
b_{i}\left(\varepsilon_{1}, \varepsilon_{2}\right)=æ_{i}\left(1-\Theta\left(\varepsilon_{1}-\varepsilon_{\mathrm{th} 1}\right) \Theta\left(\varepsilon_{\mathrm{th} 2}-\varepsilon_{2}\right)\right), i=1,2 .
$$

In (12) the parameters $æ_{i}>0$ prescribe wave attenuation in the absence of amplification mechanisms, and $\Theta(\varepsilon)$ is the Heaviside step function:

$$
\theta(\varepsilon)=\left\{\begin{array}{c}
0, \text { at } \varepsilon<0, \\
1, \text { at } \varepsilon>0
\end{array}\right.
$$

It is easy to see that, for instance, in the superposition region of tensile and compression strains, for which $b_{1,2}$ are considered equal to zero, equations (11) are satisfied by the following solution

$$
\left\{\begin{array}{l}
\varepsilon_{1,2}\left(\zeta_{1}, \zeta_{2}\right)=\left(\varepsilon_{1}\right)_{\max } \cos \left(k_{1} \zeta_{1}\right) \phi\left(\zeta_{1}, \zeta_{2}\right) \\
\varepsilon_{2}\left(\zeta_{1}, \zeta_{2}\right)=\left(\varepsilon_{2}\right)_{\max } \cos \left(k_{2} \zeta_{2}\right) \phi\left(\zeta_{1}, \zeta_{2}\right)
\end{array}\right.
$$

where $\zeta_{1}=x-v_{1} t, \zeta_{2}=y-v_{2} t$, and the function

$$
\begin{aligned}
\phi\left(\zeta_{1}, \zeta_{2}\right)= & {\left[\Theta\left(\zeta_{1}-\frac{d_{1}}{2}\right)-\Theta\left(\zeta_{1}+\frac{d_{1}}{2}\right)\right] \times } \\
& \times\left[\Theta\left(\zeta_{2}-\frac{d_{2}}{2}\right)-\Theta\left(\zeta_{2}+\frac{d_{2}}{2}\right)\right]
\end{aligned}
$$

describes the movement of the excited region with a velocity $v=v_{1}+v_{2}$ in conformity with the pattern presented in Fig.2. Thus, the formalism of the $\Theta$ - functions appears to be exceptionally convenient for reflecting the essential nonlinearity allowing for isolation of the spatial domain of the lattice which loses its stability in the course of the CWP propagation.

\section{The role of the electronic subsystem in explaining the observed features of MT}

Marked signs of the first-order transition (considerable thermal and volume effects, as well as a large temperature hysteresis between the direct and inverse transformations) 
indicate a significant deviation of the temperature $M_{s}$ during cooling from the temperature $T_{0}$ of phase equilibrium. Consequently, the process of crystal growth runs in clearly non-equilibrium conditions, and the parent phase itself, remaining metastably stable, represents an active medium able, due to the released energy, to maintain a certain threshold level of deformations carried by the CWP, sufficient for overcoming the interphase energy barrier. The notion of an active medium is in essence close to the ideology well developed for media able to generate a coherent electromagnetic radiation (the laser and maser mechanisms).

In the context of the metallic systems representing interacting subsystems of electrons and ions, it becomes natural to consider a non-equilibrium electronic subsystem as a generator of ion displacement fields (during the transformation of a part of the released energy into the energy of controlling waves). Such a photon maser effect ensures support of the amplitude level of the controlling waves that emerged at the first stage. The idea of the possibility to associate the MT process with the photon maser effect (without an understanding of the electronic subsystem's role) was first put forward in [15]. As demonstrated in [1], martensite crystal growth can be visually interpreted in the form of a switching wave, whose front has a vibrational structure playing a key role in controlling the rearrangement of the lattice which loses its stability in the region of the CWP front. In the case of transition metals (and alloys based on them), the presence of the gradients of chemical potential $\nabla \mu$ and of temperature $\nabla T$ in the region of the CWP front provides for the existence of intensive electron streams. In the stream regime there are always pairs of inversely populated states. In the transition metals that have comparatively narrow energy bands (and, correspondingly, peaks of electronic state density), the numbers of pairs $R_{\text {eff }}$ of inversely populated states within the acceptable energy range $\Delta$ in the vicinity of the Fermi level, satisfying the requirement of equidistance in terms of energy and quasi-momentum differences, may turn out to be sufficient for fulfilling the threshold conditions for wave generation:

$$
\sigma_{0}>\sigma_{\text {th }}, \quad \sigma_{\text {th }}=\hbar^{2} G_{\mathrm{d}} æ_{\mathrm{p}} /\left(W^{2} R_{\mathrm{eff}}\right),
$$

where $\sigma_{0}$ is the initial inverse population proportional to $\nabla \mu$ and $\sigma_{\text {th }}$ is the threshold value of the inverse population difference, $\hbar$ is the Planck constant, $G_{\mathrm{d}}$ and $æ_{\mathrm{p}}$ are, respectively, the attenuations of the generating $d$-electrons and generated phonons (measured in $\mathrm{rad} \cdot \mathrm{s}^{-1}$ ), $W$ is the matrix element of electron-phonons interaction. Realistic estimates of the parameters, presented in [1], demonstrate that fulfillment of (15) in iron-based alloys is possible only at $\Delta \approx 0.2 \mathrm{eV}$.

In its turn, the identification of the conditions for fulfillment of the threshold conditions of generation in a wide variation range of temperatures and concentrations of various alloying components of alloys, allows to set and solve the problem of the influence of an alloy's chemical composition on the temperature $M_{s}[1,3]$. Here, information appears, supplemental to the thermodynamic analysis, opening up a possibility to interpret the temperature $M_{\mathrm{s}}$ as the most optimal one for realization of the process of generation (or at least amplification) of the controlling waves. Consequently, the cause for a significant deviation of $M_{\mathrm{s}}$ from $T_{0}$ is clarified, and the deviation degree is specified.

A combined account of information on the optimal conditions for generation and the correlation of the characteristic spatial scales (4) allows to describe the size effect of the $M_{\mathrm{s}}$ dependence on the value of the defect-free austenite volume (in particular, on the grain diameter $D$ ). The central idea here is the need to take into account the contribution of additional scattering associated with the emergence of the initially excited state, into the complete attenuation of $s$-electrons $G$. Since the process of controlling wave generation takes place in the region of the CWP front with the transverse dimensions $d_{1,2}$, the characteristic time $\tau_{\mathrm{s}}$ for intersection of this region with $s$-electrons is expressed through their velocity $v_{\mathrm{s}}: \tau_{\mathrm{s}} \approx d_{1,2} / v_{\mathrm{s}}$. Then the estimate of the additional contribution into the attenuation can easily be found from the indeterminacy relation for energy and time. When taking into account the relation between the spatial scales (4), the contribution into the attenuation is associated with the size $L$. The population of d-states possessing their own small attenuation Gd and distributed in the energy range $\Delta \approx 0.2 \mathrm{eV}$ in the vicinity of the Fermi level $\mu$, is carried out owing to participation of strongly attenuating $s$-states, as intermediate ones. At the same time, the following relation is fulfilled

$$
G \equiv G(T, C, L) \approx 2\left|\varepsilon_{\mathrm{d}}-\mu\right|
$$

where $\left|\varepsilon_{\mathrm{d}}-\mu\right|$ characterizes the energy of generating electrons, averaged in the range $\Delta$. It is relation (16) that optimizes the connection between the physically meaningful parameters for effective generation of controlling waves in broad variation ranges of temperature and concentration. With account of (16), analytical dependencies on the physically meaningful parameters (including $L$ or within $D$ ), of the optimal generation temperature, identifiable with temperature $M_{\mathrm{s}}$, and the critical grain size $D_{c}$, below which MT is not realized down to the absolute zero temperatures, can be derived

$$
D_{c}=\frac{\hbar(L / d) v_{s}}{2\left(2\left|\varepsilon_{d}-\mu\right|-\hbar G(C)\right)} .
$$

In (17) the $s$-electron velocity $v_{\mathrm{s}} \approx 10^{6} \mathrm{~m} / \mathrm{s}$, and $G / C$ is the contribution of $s$-electrons into attenuation, conditioned by the scattering of an alloying element on ions, dependent on their concentration $C$. The value $(L / D)$, according to (4), is about $10^{2}$. Of principal interest is the conclusion about the existence of special concentrations $\mathrm{C}^{*}$ of an alloying element, approach to which is accompanied by a growth in $D_{c}$ and a decrease in $M_{s}$. Indeed, it follows from (17) that:

$$
C \rightarrow C^{*}, \quad \hbar G(C) \rightarrow 2\left|\varepsilon_{\mathrm{d}}-\mu\right|, \quad D_{\mathrm{c}} \rightarrow \infty,
$$

while $M_{\mathrm{s}} \rightarrow 0 \mathrm{~K}$ is not dependent on the size $D$. Hence, relation (17) accounts for the existence, for alloying elements, of rigid concentration boundaries, the exceeding of which blocks the process of MT. It is useful to note that the size effect was first established in [16], and the data given in [17], in our opinion, demonstrate a tendency for $D_{c}$ to grow when $C \rightarrow C^{*}$.

As fundamental consequences (and applications) of the developed theory of the size effect, we should mention the 
effect of destabilization with the strong magnetic field of an austenite preliminarily stabilized by grain refinement or severe plastic deformation [18], as well as the interpretation of the lowest temperature limit $M_{\mathrm{f}}$ for realization of MT and the evaluation of the martensite fraction formed during athermal macrokinetics of transformation (see chapter 6 in [3]). It is characteristic that the fraction of the formed martensite, which is a macroparameter, is evaluated without the use of thermodynamics. It should be noted that the effects of austenite destabilization due to an increase in the parameter $\left|\varepsilon_{\mathrm{d}}-\mu\right|$ in the magnetic field, according to (17), exhibit themselves to the maximum extent for the concentrations $\mathrm{C}$ close to $C^{*}$, when the critical value $D_{c}$ is large and decreases sharply when a strong magnetic field is turned on. The experimental data [18] for alloys of various compositions are in agreement with this conclusion.

Thus, an explicit account of the microscopic physical nature of a metallic medium experiencing a structural rearrangement in essentially non-equilibrium conditions allows to extract information inaccessible to other (phenomenological) approaches which do not take into account the system's features that are fundamental for a complete description of a rich phenomenon.

\section{On the completeness degree of the description of MT morphological features}

Currently, the achieved completeness degree of the description of the observed MT features in the developed dynamic theory for $\gamma-\alpha$ MT in iron-based alloys (in the area of its applicability) is unprecedented as compared with the existing approaches. The dynamic theory is not inferior to them in terms of accuracy, even though these approaches were specifically focused on the interpretation of the observed important facts, but knowingly neglected all the rest of the MT features.

A characteristic example is the interpretation of the observed macroscopic morphological attributes of MT, to which refer the crystallographic orientations of habits and microshear, interphase orientation ratios. Let us remind that these attributes are connected with each other in a one-to-one manner, which testifies to the presence of a single mechanism for controlling the cooperative atom displacement and represents a «visiting card» of the martensitic reaction. It is appropriate to mention that among thousands of works devoted to study of the martensite morphology, the pioneering works in terms of defining the morphological attributes are [19-21], from which [21] stands out as the most complete. The interpretation of the observed morphological attributes in the framework of the crystal-geometric approach [22-25], in spite of a number of difficulties, remained for a long time the most precise «tool» in the arsenal of phenomenological calculations.

In the framework of the wave approach, special attention was paid to the development of the theory's microscopic aspects and the description of the threshold processes, i.e. to strain levels of $10^{-4}-10^{-3}$. Therefore, only the habit plane orientation was calculated using (6). For interpretation of the rest of the attributes, it was necessary to develop an algorithm for transition from threshold to final strains, which are two or three orders of magnitude larger than threshold ones. Such an algorithm was first developed in $[26,27]$ for BCC-HCP MT. The main idea comes down to the statement: the relation between strains in the threshold regime practically does not change as the strains evolve from threshold to final values. This assertion can be viewed as a consequence of a high transformation rate and the absence of resistance from the lattice region that has lost its stability and has not yet reached a metastably stable state. But in the threshold regime the relation between strains, according to (10), is prescribed by the relation between controlling wave velocities, i.e., with a good accuracy, by the elastic constants of the parent phase. By virtue of the above stated specifics, the depth of control of the crystal formation process encompasses strains of up to 3 orders of magnitude (from the threshold to the final strain value).

Thereafter a transition was made to final strains for both non-twinned [28] and twinned $\alpha$-martensite [2]. The latter, most complicated, case required, on the one hand, an inclusion into the CWP of relatively short-wave longitudinal displacements, and on the other hand, an account of the quasi-longitudinality of long-wave displacements, whose wave vectors deviate from the symmetry axes, which leads to noticeable differences of the orientations of the principal axes of threshold strain tensors from the orientations typical for an approach of longitudinal waves. In addition, a mechanism was successfully established for the coordinated action of long-wave and short-wave displacements, closely connected with the condition of a periodic reproduction of the vibrating short-wave cell in the region of the CWP front. As a result, the match of the description of the data relating to the whole aggregate of the observed morphological attributes in the dynamic theory turned out to be at least not worse that in the crystal-geometric approach. A number of additional conclusions were obtained as well, allowing for an experimental validation.

On the basis of equation system (11) one can also naturally describe the change in the profiles of martensite crystals growing in a spatially non-uniform medium [29-31] by introducing the dependence of the "effective" wave attenuations of controlling waves on coordinates. At the present time, the achieved completeness degree of the characteristic morphology description allows to a great extent to reconstruct, on the basis of the morphological attributes, the dynamic pattern of MT development, i.e. to solve the inverse task.

\section{Concluding remarks}

A concise summary of the principal ideas that have allowed to provide an full-fledged dynamic pattern of martensite crystal formation (during cooling or rapid heating) in the limiting case of supersonic growth, demonstrates that an MT can be described with an adequate identification of the role of any hierarchical level. In other words, taken into account are the features of electron and phonon band structures, as well as the meso- and macro-levels of morphological attribute formation.

The obtained results testify about the finalization of an important stage in research, providing for the start of a 
new stage with reliably established data. These data, in our opinion, include the model of the controlled wave process, which has a great potential for solution of problems of the interaction of a growing crystal with grain boundaries and crystals that emerged earlier. Some quality scenarios, which in fact represent a program of investigations, are set forth in item 3.2 of the monograph [3]. At the same time, of undoubtful interest is also the scenario of the appearance of the initially excited state when considering the interaction of atomic displacement fluctuations in the DNC field with a grain boundary possessing its own spectrum of vibrations (see item 3.5 in [3]). There appear additional opportunities for a detailed elaboration of the features of the electronic subsystem's participation in MT in concentrated alloys. In particular, of great interest is a targeted experimental validation of the conclusions related to the existence of a critical grain size (17).

When building the dynamic theory of polytypic MT, the scheme of the controlling wave process is drastically modified, reflecting the coordinated action of longitudinal and transverse waves with collinear wave vectors. The simplest example of such a rearrangement can be an FCCHCP MT, with its quality example presented in [32]. It should be kept in mind that when coordinating the modes (especially in the short-wave range) providing for the selection of a specific variant from the spectrum of possible polytypic MT, an explicit account of the lattice discreteness and an accurate description of the frequency spectrum become essential during the construction of non-linear CWP models. Here the accumulated experience (see, e.g., [33-36]) in studying nonlinear waves in discrete media is in full measure probable to be in demand, both within an analytical description and during dynamic modeling.

The possibility of realization of MT in the nanocrystalline state constitutes a separate (in many ways, still ambiguous) area of research. Nevertheless, one can state that when the inequality $D<D_{c}$ is fulfilled, the formation of crystals with a full set of morphological attributes is unlikely, although packets of twins are still possible (see chapter 7 in [3]). The role of relatively long-wave displacements prescribing the habits in the case of $D>D_{\mathcal{c}}$ may pass to the grain's own vibrations. The outcome of this type of scenario is schematically presented in Fig.3, where the critical grain size $D_{\text {ac }}^{\prime}$ for the MT variant called accommodative $\left(D_{\text {ac }}^{\prime}<D_{c}\right)$ is indicated approximately.

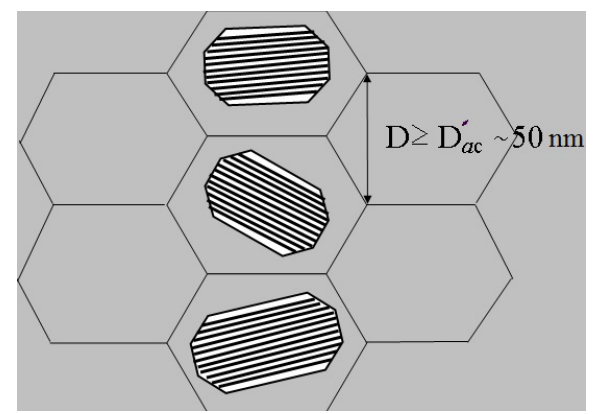

Fig. 3. Schematic illustration of an accommodative martensitic transformation in three grains, reflecting the incompleteness of the grain volume transformation, a twinned character of the transformation and the misorientation of lattices in neighboring grains.
An interesting hypothetical possibility is related to the transformation of a grain as a whole. Indeed, an atom jump to new positions in the significant part of the grain volume (necessarily including its central part where strain is maximum) with excitation of vibrations can initiate the transformation of one of the adjacent grains. Formally, to such an excited state corresponds the identical order of the sizes $L$ and $d$ in (17). But then (in the absence of the dominating elastic field of an isolated dislocation) the critical size (17) decreases approximately by two orders of magnitude. It is interesting that at typical $D_{c}$ values of around a micron (when $C$ does not yet belong to the nearest vicinity of the special concentration $C^{*}$ ) the condition $\mathrm{L} / \mathrm{d} \sim 1$ in (17) leads to a quite reasonable evaluation of the magnitude order of $D_{\mathrm{ac}} \sim 10 \mathrm{~nm}$ for the critical grain size in respect to the "single-crystal-to-singlecrystal" rearrangement (the designation $D_{\text {ac }}$ is introduced to distinguish the critical size of a uniformly transformed grain from the size $D_{\text {ac }}$ for a grain with a microscopic twinned volume). Such rearrangements are probably observed, but at smaller, than in the case of the $\gamma-\alpha \mathrm{MT}$ in iron-based alloys, levels of threshold and final strains. For instance, in the context of the B2-B19 transition in the $\mathrm{Ti}_{50} \mathrm{Ni}_{25} \mathrm{Cu}_{25}$ alloy the finishing single crystal rearrangements were recorded [37], even though without a clarification of the rearrangement mechanism details. According to the experimental data [37] on the dependence $M_{s}(D)$, it follows that the transformation is blocked at grain sizes below $\approx 20 \mathrm{~nm}$, to which $M_{\mathrm{s}} \approx 300 \mathrm{~K}$ corresponds. Since the indicated value of $M_{\mathrm{s}}$ is still far from the absolute zero, the size $\approx 20 \mathrm{~nm}$ exceeds the critical value of $D_{\mathrm{ac}}$, for which, by definition, $M_{\mathrm{s}}\left(D_{\mathrm{ac}}\right)=0 \mathrm{~K}$ is fulfilled. Nevertheless, not applying a strict processing of the data [37], we should note that $D_{\text {ac }}=20 \mathrm{~nm}$ can be obtained at $L / d=2$. It is evident that to such a choice of the $L / d$ parameter value corresponds the transformation, as a whole one, of only the central grain part with a size $d$ constituting a half of $L$ (of the grain diameter $D_{a c}$ ). This interpretation is in good agreement with the variant, mentioned in [3], of a transformation of the central part with a well expressed crystalline structure different from a less perfect structure of the near-boundary region, constituting a significant part of the grain volume (see Fig.4).

Besides, as noted above, the largest strain during grain vibrations is attained in the grain center, turning into zero at its boundary. Consequently, fulfillment of the requirement

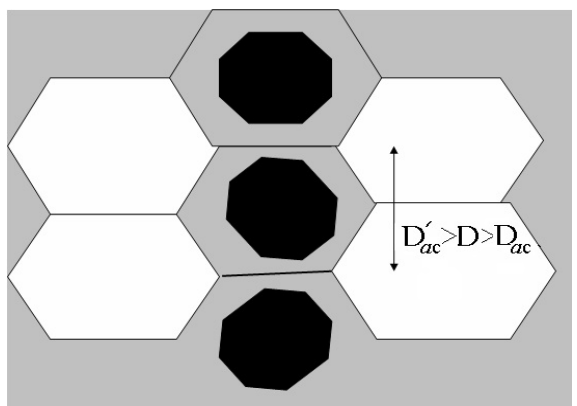

Fig. 4. Schematic illustration of an accommodative martensitic transformation in three nano-grains, reflecting the incompleteness of the grain volume transformation. The regions inside the grains that have transformed as a whole are conventionally marked by the black color. 
$d<\lambda / 2$ (see item 2) is quite similar to the inequality $d<L$. Of course, without clearing up the mechanism of MT realization, it is premature to make any final statements, since a «singlecrystal-to-single-crystal» rearrangement is possible also due to a relatively slow movement of the interphase boundary in the case of a thermoelastic character of MT. It should also be noted that the thermoelastic growth during the movement of «transformation dislocations» can increase also the sizes of the central region with a diameter $\mathrm{d}$, which at first experienced a jump-like rearrangement. Hence, hypothetically possible are at least three scenarios of single-crystal transformation of a significant grain volume fraction.

It is appropriate to mention that, alongside with spontaneously running MT (at a change in the parent phase temperature), one distinguishes also MT at an applied external elastic stress and MT running in the conditions of plastic flow. The corresponding transformation products are referred to as «stress-induced martensite» and «strain-induced martensite». The mechanism of «stress-induced martensite» formation is similar to "cooling-induced martensite», only the number of the observed crystallographic orientations (in accordance with the symmetry of the external stress field) is reduced, i.e. «stress-induced martensite» can form with a supersonic rate (in relation to longitudinal waves). The formation of «strain-induced martensite» crystals (as well as the shear bands) at moderate plastic strain rates, in our opinion, is quite adequately described in the framework of the cryston approach (see, for instance, [38-39]). In this case the rate of martensite crystal formation at the temperature $M_{\mathrm{d}}>M_{\mathrm{s}}$ should not exceed the velocity of transverse elastic waves. And if the external loading is effected through a shock wave, the limitation of "strain-induced" martensite crystal growth rate by the velocity of transverse waves is not obligatory.

It should be stressed that the concept, introduced in the framework of a new paradigm, of the initially excited state (IES) turns out to be constructive, also during solution of problems related to account of the effect of the carbon diffusion process on the spatial scale $d$ determining the transverse dimension of IES.

For instance, the fulfillment of the condition of diffusion suppression on the scale $\mathrm{d}$ allows for a natural evaluation of the critical rates of austenite cooling [40] required for a transition to the lamellar morphology of martensite in some alloyed steels [41]. Conversely, the characteristic incubation times during isothermal holding of austenite (overcooled to the temperature $B_{\mathrm{s}}>M_{\mathrm{s}}$ ), preceding to a rapid growth of lath crystals of bainitic ferrite, are conditioned by carbon diffusion on the spatial scale $d$. As a consequence, the order of the "effective" growth rate of a bainitic ferrite macroplate is prescribed by a correlation of the mean size of laths comprising the macroplate (and forming at a supersonic rate) to the mean incubation time between consecutive starts of lath crystal growth [42]. Thus, an additional wide scope is opened up for experimental and theoretical studies of structural transformation, combining the alternating stages of diffusion activity and diffusionless wave growth.

Drawing up a summary, the authors would like to thank the journal's editorial board for their offer to present a brief overview of our original results. We also hope that we have managed to set forth the concepts that transparently disclose the physical nature of the limiting case of martensite crystals' supersonic formation, and attract attention to a number of tasks for initiation of new investigations in the topical thematic area of martensitic and bainitic transformations. Currently, the dynamic theory is actively developing, finding a successful application in all the above-mentioned areas (see, e.g., [43-51]). It is useful to bear in mind that in the methodological aspect this review should be viewed as mutually complementary with the reviews [52] and [53].

This work was performed with financial support from the RFBR (project no. 14-08-00734).

\section{References}

1. M. P. Kashchenko, V.G. Chashchina. Wave Model of Martensite during $\gamma$ - $a$ Transformation in Iron Based Alloys. 2nd. Moscow - Izhevsk, NITS «Regular and Chaotic Dynamics», Izhevsk Institute of Computer Science. (2010) 280 p.

2. M.P. Kashchenko, V.G. Chashchina. UGLTU. Ekaterinburg. (2009). (in Russian)

3. M.P. Kashchenko, V.G. Chashchina, Dynamic Theory of $\gamma$-a Martensitic Transformation in Iron Alloys and Solution of the Problem of Critical Grain Size (Moscow Izhevsk, NITS «Regular and Chaotic Dynamics», Izhevsk Institute of Computer Science. (2010) 132 p.

4. N. Ya. Selyakov, G. V. Kurdyumov, N.T. Gudtsov, Zh. Prikl. Fiz. 2, 51 (1927).

5. G. V. Kurdyumov, L.M. Utevskii, R.I. Entin. Transformations in Iron and Steel. Nauka, Moscow. (1977). (in Russian)

6. R. F. Bunsha, R. F. Mehl. Trans. AIME. 197. 1251 (1953).

7. F.L. Lokshin. Nauchnie Dokladi Visshei Shkoli. 2, 205 (1958). (in Russian)

8. Yu.I. Meshcheryakov, M.P. Kashchenko, V.B. Vasilkov, S. A. Atroshenko, JTP Letters, 19(2), 75 (1993).

9. F. I. Fedorov. Theory of Elastic Waves in Crystals. Moscow, Nauka. (1965) 388p. (in Russian)

10. M.P. Kashchenko et al. The Physics of Metals and Metallography. 67, 146 (1992).

11. V.V. Letuchev, S. V. Konovalov, S. V. Neskoromnyi, M.P. Kashchenko. Journal of Materials Science Letters. 24, 1683 (1992).

12. M.P. Kashchenko et al. Izvestiya RAS. Metals. 2, 105 (1992). (in Russian)

13. M.P. Kashchenko et al. The Physics of Metals and Metallography. 76, 90 (1993).

14. U. Kayser. J. Phys. France. 2, 60 (1972).

15. M.P. Kashchenko, V.G. Chashchina, S. V. Vikharev The Physics of Metals and Metallography.110(4), 305 (2010).

16. E. Scheil Z. Anorg. Chem. 180, 1 (1929).

17. M. Umemoto, W.S. Owen. Metallurgical transactions. 5, 2041 (1974).

18. V.M.Schastivtsev, Yu. V.Kaletina, E. A. Fokina. Martensitic Transformation in Magnetic Field. Ekaterinburg Ural branch of RAS. (2007) 322 p. (in Russian)

19. G. Kurdyumov, G. Sachs. Z. Phys. 9-10, 165 (1930).

20. Z. Nishiyama. Sci. Rpts. Tohoku Imp. Univ. 23, 637 (1934). 
21. A.B. Greninger, A.R. Troiano. Metals Transactions. 185, 590 (1949).

22. M.S. Wechsler, D. S. Lieberman, T.A. Read. Trans. AIME. 197, 1503 (1953).

23. J.S. Bowles, J.K. Mackenzie. Acta Metallurgica. 2, 129 (1954).

24. Bowles J S, Mackenzie J K Acta Metallurgica. 2, 138 (1954).

25. Bowles J S, Mackenzie J K Acta Metallurgica. 2, 224 (1954).

26. M.P. Kashchenko, V.G. Chashchina. The Physics of Metals and Metallography. 105, 571 (2008).

27. M.P. Kashchenko, V.G. Chashchina. The Physics of Metals and Metallography. 106, 16 (2008).

28. V. G. Chashchina. Russian Physics Journal. 7, 95 (2009).

29. M. P. Kashchenko, V. G. Chashchina, S. V. Vikharev. Metal Science and Heat Treatment. 52(7-8), 357 (2010).

30. V. Chashchina, M. Kashchenko, S. Vikharev. ArXiv: cond-mat 1003.2952(3) (2010) 5p.

31. M.P. Kashchenko, V.G. Chashchina, S. V. Vikharev. Fundamental Problems and Modern Technologies of Materials Science. 7(1), 7 (2010).

32. V.G. Chaschina. Russian Physics Journal. 52(7), 766 (2009).

33. S. V. Dmitriev, T. Shigenari, S. M. Volkova, A. A. Vasiliev, K. Abe. Comp. Mater. Sci. 13, 227 (1999).

34. S. V. Dmitriev. Ferroelectrics. 349, 33 (2007).

35. Y. V. Bebikhov, S. V. Dmitriev, S. V. Suchkov, A. Khare. Phys. Lett. A374, 1477 (2010).

36. I. Roy, S. V. Dmitriev, P.G. Kevrekidis, A. Saxena. Phys. Rev. E76, 026601 (2007).

37. N. N. Kuranova, PhD Thesis. Ekaterinburg. (2009) 173p.

38. M.P. Kashchenko, V.G. Chashchina, A.G. Semenovih, Physical Mesomechanics. 6(3), 37 (2003).
39. M.P. Kashchenko, V.G. Chashchina, A.G. Semenovih. Physical Mesomechanics. 6(1), 95 (2003).

40. M.P. Kashchenko, V.G. Chashchina. The Physics of Metals and Metallography. 113, 894 (2012).

41. D. A. Mirzaev, M. M. Shteinberg, T. N. Ponomareva, and V. M. Schastlivtsev. Fiz. Met. Metalloved. 47, 986 (1979).

42. M.P. Kashchenko, V.G. Chashchina. The Physics of Metals and Metallography. 114, 266 (2013).

43. M.P. Kashchenko, K. N. Dzhemilev, V.G. Chashchina. Russian Physics Journal. 55, 1052 (2013).

44. M.P. Kashchenko, K. N. Dzhemilev, V.G. Chashchina. Russian Physics Journal. 55, 1235 (2013).

45. M.P. Kashchenko, V.G. Chashchina. Russian Physics Journal. 56, 557 (2013).

46. M.P. Kashchenko, V.G. Chashchina. The Physics of Metals and Metallography. 114, 821 (2013).

47. M.P. Kashchenko, V.G. Chashchina. Russian Physics Journal. 56, 647 (2013).

48. M.P. Kashchenko, V.G. Chashchina. The Physics of Metals and Metallography. 114, 977 (2013).

49. M.P. Kashchenko, V.G. Chashchina. Russian Physics Journal. 57, 1099 (2014).

50. M.P. Kashchenko, V.G. Chashchina. Metal Science and Heat Treatment. 56, 343 (2014).

51. M.P. Kashchenko, V.G. Chashchina. Metal Science and Heat Treatment. 56, 643 (2014).

52. M.P. Kashchenko, V.G. Chashchina. UFN. 181(4), 345 (2011). [Translated from Physics -Uspekhi, 54(4) (2011)]. (in Russian)

53. M.P. Kashchenko, V.G. Chashchina. Materials Science Forum. European symposium on martensitic transformations (ESOMAT 2012). 738-739, 3 (2013). 